\title{
On Arithmetical Truth of the Self-Referential Sentences
}

\author{
KaAve Lajevardi \& Saeed Salehi
}

\begin{abstract}
We take an argument of Gödel's from his ground-breaking 1931 paper, generalize it, and examine its validity. The argument in question is this: the sentence $G$ says about itself that it is not provable, and $G$ is indeed not provable; therefore, $G$ is true.
\end{abstract}

\section{Introduction}

As is well known, Gödel begins his 1931 masterpiece [2] with an introductory section, Section 1, wherein he explains the main ideas behind the (first) incompleteness theorem in an informal and intuitive way, with an explicit caveat that those remarks are being made "without any claim to complete precision". What he does in that section is, among other things, to introduce, in a very lucid way, the idea of encoding the syntax and that of diagonalization.

But Gödel's introduction is not confined to what can be found, more formally expressed, in the technical parts of the paper. Gödel's official statement of the first incompleteness theorem (his Theorem VI) asserts that for every theory satisfying certain conditions, there is a sentence, now called the Gödel sentence of the theory, which is expressible in the language of the theory but is neither provable nor refutable in the theory-the theorem does not talk about the truth of that undecidable sentence. In his Section 1, however, Gödel chooses to also talk about the truth of the Gödel sentence of the system of Principia Mathematica, and observes that the proof of the first incompleteness theorem, as presented in that section, is similar to Richard's paradox. Whatever expository merits it might have, this move is a problematic one, and Gödel shows awareness of this. He says that his method of proof is applicable to any formal system that has two conditions, the second of which being "every provable formula is true in the interpretation considered" ([2, page 151] emphasis added). He then writes, "The purpose of carrying out the above proof with full precision in what follows is, among other things, to replace the second of the assumptions just mentioned by a purely formal and much weaker one" (ibid, emphasis added). This "much weaker" condition, called $\omega$-consistency by Gödel, is one of the points of focus in our discussion (note that $\omega$-consistency is stronger than [simple] consistency, see e.g. [4, Theorems 14,15]). 
Our purpose in this note is not to philosophize about the notion of truth or doing exegetical work on its rôle in Gödel's classic paper. Rather, we wish to draw attentions to an informal argument, to the effect that the Gödel sentence of the system Principia Mathematica is true (that is, true in $\mathbb{N}$ ), which is presented by Gödel in his introductory section. The argument in question goes as follows [2, p. 151] (italics in the original):

From the remark that $[R(q) ; q]$ says about itself that it is not provable, it follows at once that $[R(q) ; q]$ is true, for $[R(q) ; q]$ is indeed unprovable (being undecidable).

Here $[R(q) ; q]$ is what is now called the Gödel sentence of a theory (or "system") which is subject to the first incompleteness theorem (that can be taken as Peano's Arithmetic PA or its recursively axiomatizable $\omega$-consistent extensions). This is nowadays denoted by $G$, which is by definition a sentence $P$ that is equivalent to $\neg \operatorname{Pr}(\# P)$ (also provably so), where $\operatorname{Pr}$ is the provability predicate of the theory and \#P is the standard term for the Gödel number of $P$.

As we understand the above passage, its logical form is the following, where $A$ is an arbitrary sentence expressible in the language of the theory:

(1) $A$ says about itself that it has a property $F$.

(2) $A$ indeed has the property $F$.

(3) Therefore: $A$ is true.

That Gödel says that it follows at once (Gödel's term "sofort" could also be translated to immediately) that $G$ is true suggests that, in Gödel's view, we are not dealing with an enthymeme-it seems to us that, for Gödel, the argument scheme has no missing premises. It is our task in the next section to argue that the (1)-(3) argument scheme is invalid, that is to say, there are situations wherein the premises are true while the conclusion false. Naturally enough, the validity of the argument hinges on its terms, in particular on what it is for a sentence to be "true", and what is meant by a sentence "saying of itself" that it has a certain property. As for the first term, it is almost obvious from Gödel's introductory section that, like many modern writers in mathematical logic, when he writes "true" simpliciter, he means true in the standard model, $\mathbb{N}$ (thus [2]'s footnote 4 on page 145: “... no other notions occur but + (addition) and - (multiplication), both for natural numbers, and in which the quantifiers ( $x$ ), too, apply to natural numbers only.") Regarding the notion of saying something of oneself, we shall consider two interpretations that might be ascribed to Gödel.

\section{The Invalidity of the Generalized Argument}

How are we to understand the expression " $A$ says that it has the property $F$ "? Modulo an agreement over the meaning of "holding", to which we shall return shortly, we find it quite plausible to think that if the conditional $A \longrightarrow F(\# A)$ holds, then $A$ says, inter 
alia, that $A$ has property $F$. So, if we take "holding" as "being true in the standard model of natural numbers $\mathbb{N}$ " and "saying" as "implying", then the argument is not valid: if we take $\gamma$ to be the sentence $G \wedge(0=1)$ then both of the sentences $\gamma \rightarrow \neg \operatorname{Pr}(\# \gamma)$ and $\neg \operatorname{Pr}(\# \gamma)$ hold, but $\gamma$ does not hold. Thus to say that $A$ says, exactly, that $A$ has property $F$ must be something stronger, to wit that the biconditional $A \longleftrightarrow F(\# A)$ holds. And this is, in fact, what is taken by some authors (including Gödel) to be the meaning of " $A$ says of $A$ that it has property $F$ " (see e.g. [3, 6] and references therein).

As for the meaning of "holding", we already presented evidence for the claim that what Gödel meant by it is being true in the standard model, $\mathbb{N}$. However, let us recognize another reading of it-which is also suggested in the literature (see [3, 6] and references therein) - namely being provable in a given theory. Having fixed a theory $T$ (which we suppose to be an $\omega$-consistent, recursively axiomatizable extension of Peano's Arithmetic $P A$ ), we then have eight possible ways of interpreting the (1)-(3) argument scheme. Here is the complete list, of which we find (VII), (V), and (III) the most interesting:

$$
\begin{gathered}
\text { (I) } \frac{\mathbb{N} \vDash A \leftrightarrow F(\# A), \quad \mathbb{N} \vDash F(\# A)}{\mathbb{N} \vDash A} \quad \text { (II) } \frac{\mathbb{N} \vDash A \leftrightarrow F(\# A), \quad \mathbb{N} \vDash F(\# A)}{T \vdash A} \\
\text { (III) } \frac{\mathbb{N} \vDash A \leftrightarrow F(\# A), \quad T \vdash F(\# A)}{\mathbb{N} \vDash A} \quad \text { (IV) } \frac{\mathbb{N} \vDash A \leftrightarrow F(\# A), \quad T \vdash F(\# A)}{T \vdash A} \\
\text { (V) } \frac{T \vdash A \leftrightarrow F(\# A), \quad \mathbb{N} \vDash F(\# A)}{\mathbb{N} \vDash A} \quad \text { (VI) } \frac{T \vdash A \leftrightarrow F(\# A), \quad \mathbb{N} \vDash F(\# A)}{T \vdash A} \\
\text { (VII) } \frac{T \vdash A \leftrightarrow F(\# A), \quad T \vdash F(\# A)}{\mathbb{N} \vDash A} \quad \text { (VIII) } \frac{T \vdash A \leftrightarrow F(\# A), \quad T \vdash F(\# A)}{T \vdash A}
\end{gathered}
$$

Of these, (I) and (VIII) are of course valid because of the truth-condition of the material conditional and Modus Ponens, respectively. For all other cases, we will present triples $(A, F, T)$ which invalidate them.

Theorem 2.1 The argument (IV) is invalid for $A=G, F(x) \equiv(x=\# G)$, and $T=P A$.

Proof. Obviously, $\mathbb{N} \vDash F(\# G)$ and $T \vdash F(\# G)$. By Gödel's proof $\mathbb{N} \vDash G$ holds and so $\mathbb{N} \vDash G \leftrightarrow F(\# G)$. On the other hand, by Gödel's theorem, $T \nvdash G$.

Theorem 2.2 The arguments (II) and (VI) are invalid for $A=G, F(x) \equiv \neg \operatorname{Pr}(x)$, and $T=P A$.

Proof. We already have $\mathbb{N} \vDash G \leftrightarrow \neg \operatorname{Pr}(\# G)$. Since by Gödel's theorem we have $P A \nvdash G$, it follows that $\neg \operatorname{Pr}(\# G)$ is true, i.e., $\mathbb{N} \vDash \neg \operatorname{Pr}(\# G)$. 
Of course the arguments (III), (V) and (VII) are all valid if $T$ is a sound theory (i.e., when $\mathbb{N} \vDash T$ ). As we mentioned in the Introduction, Gödel replaces the soundness condition with the much weaker condition of $\omega$-consistency. As Isaacson mentions in [4], Gödel states in [2] that the notion of $\omega$-consistency is "much weaker" than soundness but gives no argument for this claim. It is shown in [4, Proposition 19] (with a proof attributed to Kreisel in the 1950s) that there exists a false sentence $K$ such that the theory $P A+K$ is $\omega$-consistent. Also, the sentence $K$ is a diagonal sentence of a formula $H(x)$; i.e., the equivalence $K \leftrightarrow H(\# K)$ holds (is $P A$-provable and true in $\mathbb{N}$ ).

Theorem 2.3 The argument $(\mathrm{V})$ does not hold for $A=K, F(x) \equiv(x=\# K)$, and $T=P A+K$.

Proof. By $P A \vdash F(\# K)$ we have $P A+K \vdash K \leftrightarrow F(\# K)$. Now, $\mathbb{N} \vDash F(\# K)$ holds trivially. By [4, Propositions 19], $\mathbb{N} \not \models K$.

Theorem 2.4 Neither (III) nor (VII) hold for $A=K, F(x) \equiv H(x)$, and $T=P A+K$.

Proof. We already have $P A \vdash K \leftrightarrow H(\# K)$ and $\mathbb{N} \vDash K \leftrightarrow H(\# K)$ by definition, and so $P A+K \vdash K \leftrightarrow H(\# K)$ holds too. The latter also implies that $P A+K \vdash H(\# K)$. Finally, by [4, Propositions 19] we have $\mathbb{N} \not \models K$.

Let us acknowledge the fact that perhaps it is only by overgeneralizing Gödel's informal argument that we are making it invalid. Had we not abstracted from the specific properties of $A, F$, and $T$, Gödel's informal argument would be valid, even in the interesting cases of (III), (V), and (VII) (though it would lose much of its appeal). This is substantiated in the following:

Proposition 2.5 If $A, F$ are both $\Pi_{1}$ and $T$ is an $\omega$-consistent extension of $P A$, then the arguments (III), (V), and (VII) are valid.

Proof. If $A, F(x) \in \Pi_{1}$ then $F(\# A)$ and $A \leftrightarrow F(\# A)$ are both $\Sigma_{2}$. By [4, Theorem 17], all the $T$-provable $\Sigma_{2}$-sentences are true. So, the provability of $F(\# A)$ or $A \leftrightarrow F(\# A)$ in $T$ implies their truth. Whence, (III), (V), and (VII) all reduce to (I).

\section{Back to the Truth of the Gödel Sentence}

The sentence $G$ is called the Gödel sentence of the theory in consideration (say, of $P A$ ). One probable rationale for this is that if $G^{\prime}$ is any other sentence that is equivalent to its unprovability, that is $G^{\prime}$ is equivalent to $\neg \operatorname{Pr}\left(\# G^{\prime}\right)$, then $G$ and $G^{\prime}$ are equivalent (see e.g., [5]). But this is not a convincing reason when the theory is not sound:

Remark 3.1 Let $S=P A+\neg \operatorname{Con}(P A)$, where $\operatorname{Con}(P A)$ is the consistency statement of $P A, \neg \operatorname{Pr}(\#[0=1])$. By Gödel's second incompleteness theorem the theory $S$ is consistent (but not sound). We show that for any true $\Sigma_{1}$-sentence $\sigma$, the sentence $\sigma^{\prime}$ which is defined 
to be $\operatorname{Con}(S) \wedge \sigma$, is equivalent to its unprovability in $S$; later we also show that this holds for any false $\Pi_{1}$-sentence $\varrho$ too.

To see this take $\sigma$ to be any true $\Sigma_{1}$-sentence. Then by Löb's Theorem we have $S \vdash \operatorname{Con}(S) \rightarrow \neg \operatorname{Pr}_{S}(\# \operatorname{Con}(S))$ and by the $\Sigma_{1}$-completeness, $S \vdash \sigma$; so

$$
S \vdash \operatorname{Con}(S) \wedge \sigma \rightarrow \neg \operatorname{Pr}_{S}(\#[\operatorname{Con}(S) \wedge \sigma]) .
$$

On the other hand $S \vdash \neg \operatorname{Pr}_{S}(\# \psi) \rightarrow \operatorname{Con}(S)$, for any $\psi$, which, together with $S \vdash \sigma$, implies the derivability of the converse implication

$$
S \vdash \neg \operatorname{Pr}_{S}(\#[\operatorname{Con}(S) \wedge \sigma]) \rightarrow \operatorname{Con}(S) \wedge \sigma .
$$

Thus, $\sigma^{\prime}=\operatorname{Con}(S) \wedge \sigma$ is equivalent to its unprovability in $S: S \vdash \sigma^{\prime} \leftrightarrow \neg \operatorname{Pr}_{S}\left(\# \sigma^{\prime}\right)$.

Now, take $\varrho$ to be a false $\Pi_{1}$-sentence. Then $\mathbb{N} \vDash \neg \varrho$ and $\neg \varrho \in \Sigma_{1}$; so $S \vdash \neg \varrho$. Also, $S \vdash \neg \operatorname{Con}(S)$ (because $S \vdash \neg \operatorname{Con}(P A)$ and $P A \subset S$ ); whence $S \vdash \operatorname{Pr}_{S}(\# \theta)$ for any $\theta$ and in particular $S \vdash \operatorname{Pr}_{S}(\# \varrho)$. So, $S \vdash \varrho \leftrightarrow \neg \operatorname{Pr}_{S}(\# \varrho)$ which shows that $\varrho$ is equivalent to its unprovability in $S$.

It can be easily seen that none of the true sentences $\sigma^{\prime}=\operatorname{Con}(S) \wedge \sigma$ (where we have $\mathbb{N} \vDash \sigma \in \Sigma_{1}$ ) is equivalent to any of the false $\Pi_{1}$-sentences $\varrho$ (where $\mathbb{N} \not \models \varrho$ ), even though they are all provably equivalent inside the theory $S$.

Thus, a sentence which is equivalent to its unprovability inside a theory, does not deserve to be called "the Gödel sentence" of that theory. A better definition could be:

Definition 3.2 The Gödel sentence of a theory $T$ is a sentence $P$ such that

$$
\text { (i) } T \vdash P \leftrightarrow \neg \operatorname{Pr}_{T}(\# P) \text { and also (ii) } \mathbb{N} \vDash P \leftrightarrow \neg \operatorname{Pr}_{T}(\# P) \text {. }
$$

All such sentences are equivalent (in $\mathbb{N}$ and provably in $T$ if $T \supseteq P A$ ).

Now, by this definition one can show that the Gödel sentence(s) of a theory is(are) true if and only if the theory is consistent (cf. [4, Theorems 10,11]):

Theorem 3.3 Let $P$ be the Gödel sentence of a theory $T$. Then $P$ is true if and only if $T$ is consistent.

Proof. If $P$ is true then, by Definition 3.2 (ii), so is $\neg \operatorname{Pr}_{T}(\# P)$ thus $T$ is consistent; and if $T$ is consistent then it cannot prove $P$ (otherwise if $T \vdash P$ then on the one hand by Definition 3.2 (i) $T \vdash \neg \operatorname{Pr}_{T}(\# P)$ and on the other hand $\operatorname{Pr}_{T}(\# P)$ is a true $\Sigma_{1}$-sentence and so is provable in $T$ which contradicts the consistency of $T$ ) and so $\neg \operatorname{Pr}_{T}(\# P)$ is true whence, by Definition 3.2 (ii), $P$ is true.

It has been argued in the literature (see e.g. [1] or [7] and references therein) that since the consistency of the theory implies (and moreover is equivalent to) its Gödel's sentence(s), even provably so inside the theory, then for "seeing " the truth of the Gödel sentence(s) the consistency of the theory should be seen. Let us note, that a sentence which is equivalent to its own provability inside the theory is not thereby (equivalent to) the Gödel sentence(s) of the theory. In Remark 3.1 we had $S \vdash \operatorname{Con}(S) \leftrightarrow \varrho$ for any false $\Pi_{1}$-sentence $\varrho$ (and also $S \vdash \varrho \leftrightarrow \neg \operatorname{Pr}_{S}(\# \varrho)$ ) but $\operatorname{Con}(S)$ is a true $\Pi_{1}$-sentence while $\varrho$ is not. Our last result provides a necessary and sufficient condition for the truth of all the $\Pi_{1}$-sentences that are equivalent to their unprovability inside the theory. 
Theorem 3.4 For a recursively axiomatizable extension $T$ of $P A$, all of the $\Pi_{1}$-sentences $\theta$ which satisfy $T \vdash \theta \leftrightarrow \neg \operatorname{Pr}_{T}(\# \theta)$ are true if and only if $T+\operatorname{Con}(T)$ is consistent.

Proof. If $T+\operatorname{Con}(T)$ is not consistent then $T \vdash \neg \operatorname{Con}(T)$ and so, $T \vdash \operatorname{Pr}(\# \psi)$ for any $\psi$. Also, for any false $\Pi_{1}$-sentence $\varrho$ we have $T \vdash \neg \varrho$. Whence, $T \vdash \varrho \leftrightarrow \neg \operatorname{Pr}_{T}(\# \varrho)$ holds which shows that any false $\Pi_{1}$-sentence is equivalent to its unprovability in $T$. Suppose now that a false $\Pi_{1}$-sentence $\theta$ satisfies $T \vdash \theta \leftrightarrow \neg \operatorname{Pr}_{T}(\# \theta)$. Then $\neg \theta$ is a true $\Sigma_{1}$ sentence, whence $T \vdash \neg \theta$. This, by $T \vdash \theta \leftrightarrow \operatorname{Con}(T)$, implies that $T \vdash \neg \operatorname{Con}(T)$ and so the theory $T+\operatorname{Con}(T)$ is not consistent.

The consistency of $T+\operatorname{Con}(T)$ is a strictly stronger condition than the simple consistency of $T$ (see [4, Corollary 37]), while $\omega$-consistent theories satisfy this condition (see [4, Theorem 36]). Thus if a $\Pi_{1}$-sentence says, inside a consistent theory, that it is not provable in that theory, this, in itself, is no reason for believing that that sentence is true (cf. Remark 3.1) unless that theory is also consistent with its own consistency statement. Finally, let us note that the consistency of $T+\operatorname{Con}(T)$ is a necessary and sufficient condition for the independence (unprovability and unrefutability) of the Gödel sentences from the theory (see [4, Theorem 35]).

\section{Conclusion}

Gödel's original argument was this: the sentence $G$ says that it is not provable in $T$, and $G$ is indeed not provable in $T$; thus $G$ is true. A necessary condition for the validity of this argument is the consistency of $T$, see Theorem 3.3, note that here $G$ does not need to be a $\Pi_{1}$-sentence. If $G$ says (only) inside the theory that it is not $T$-provable (and does not say so in the real world, i.e., $T \vdash G \leftrightarrow \neg \operatorname{Pr}_{T}(\# G)$ but $\mathbb{N} \not \models G \leftrightarrow \neg \operatorname{Pr}_{T}(\# G)$ ), then $G$ need not be true, see Remark 3.1 as $T$ might not be sound (and so $T$ could be lying about $G$ or what it says!). So, Gödel's argument is invalid in this case, even if $G$ is a $\Pi_{1}$-sentence. But it is valid if $G$ is a $\Pi_{1}$-sentence and if $T$ is $\omega$-consistent (as Gödel wanted the theory to be); see Theorem 3.4 and the explanations after its proof. Over-generalizing the argument proved it to be invalid (in six out of the eight possible cases), whether we take "saying" to be 'inside the theory' or 'in the real world' $(\mathbb{N}$ ), and "holding" as 'being true' (in $\mathbb{N}$ ) or 'being provable' (in an $\omega$-consistent, recursively axiomatizable extension of Peano's Arithmetic); see Theorems 2.1, 2.2, 2.3 and 2.4. However, the argument remains valid for $\Pi_{1}$-sentences and $\Pi_{1}$-properties, see Proposition 2.5. as they were in Gödel's case [2]. Our (I)_-(VIII) rules are overgeneralizing Gödel's argument, since he would not mean "the unprovability of $G$ " to be provable in the theory, because by the second incompleteness theorem (which was intended to be proved in the never-written second part of [2]) the consistency is not provable in the theory and so it cannot prove the unprovability of anything; however, in the rules (III), (IV), (VII) and (VIII) we assumed the $F(\# A)$ to be provable in the theory. 


\section{References}

[1] George Boolos, On "seeing" the Truth of the Gödel Sentence, Behavioral and Brain Sciences 13:4 (1990) 655-656. DOI: 10.1017/S0140525X00080687. Also reprinted in: Logic, Logic and Logic (ISBN: 9780674537675), R. Jeffrey (ed.), Harvard University Press (1999), pp. 389-391.

[2] KURT GöDEL, Über formal unentscheidbare Sätze der Principia Mathematica und verwandter Systeme, I., Monatshefte für Mathematik und Physik 38:1 (1931) 173198. DOI: 10.1007/BF01700692. Translated as “On Formally Undecidable Propositions of Principia Mathematica and Related Systems, I.”, in: Kurt Gödel Collected Works, Volume I: Publications 1929-1936 (ISBN: 9780195039641), S. Feferman et al. (eds.), Oxford University Press (1986), pp. 135-152.

[3] Volker Halbach \& Alber Visser, Self-reference in Arithmetic, I, The Review of Symbolic Logic 7:4 (2014) 671-691. DOI: 10.1017/S1755020314000288. Selfreference in Arithmetic, II, ibid 692-712. DOI: 10.1017/S175502031400029X.

[4] Daniel IsAacson, "Necessary and Sufficient Conditions for Undecidability of the Gödel Sentence and its Truth”, in: Logic, Mathematics, Philosophy: Vintage Enthusiasms_Essays in Honour of John L. Bell, D. DeVidi \& M. Hallett \& P. Clarke (eds.), Springer (2011), pp. 135-152. DOI: 10.1007/978-94-007-0214-1_7.

[5] Per Lindström, Provability Logic—a short introduction, Theoria 62:1-2 (1996) 19-61. DOI: 10.1111/j.1755-2567.1996.tb00529.x.

[6] Peter Milne, On Gödel Sentences and What They Say, Philosophia Mathematica 15:2 (2007) 193-226. DOI: 10.1093/philmat/nkm015.

[7] Panu RaAttkainen, On the Philosophical Relevance of Gödel's Incompleteness Theorems, Revue Internationale de Philosophie 59:4 (2005) 513-534. URL: https://www.cairn.info/load_pdf.php?ID_ARTICLE=RIP_234_0513 